\title{
Oculo-orofaciodigital defect with endocrinopathy in Joubert syndrome: A rare combination
}

Joubert syndrome (JS) is a rare autosomal recessive disorder with key finding of molar tooth sign (MTS) on axial magnetic resonance images (MRI). Six phenotypic subgroups are known in JS: Pure JS, JS with ocular defect, JS with renal defect, JS with oculo renal defects, JS with hepatic defect, and JS with orofaciodigital defects. We are reporting a case of 14-year-old boy, who presented with oculoorofaciodigital defect (polydactyly, syndactyly, ankyloglossia, ptosis, retinal dystrophy) with endocrinopathy (gynecomastia, micropenis, low testosterone) and MRI suggestive of MTS. Such kind of oculo-orofaciodigital defect with endocrinopathy (without puebarche, thalarche and menarche until the age of 17 years with hormonal disturbance) was also found in his elder sister. JS with endocrinopathy is a rare presentation, this is either expansion of the spectrum of JS in Indian people or a combination with endocrinopathy can evolve in later age.

Key words: Endocrinopathy, joubert, molar tooth sign, oculo-orofaciodigital

\section{INTRODUCTION}

The prevalence of Joubert syndrome (JS) is at least 1:100,000 world-wide ${ }^{[1-3]}$ may be even higher, not each JS patient has to be reported. Combinations of various phenotypic subgroups of JS have been highlighted previously from other part of the world. We are reporting the two siblings from Western Rajasthan of India who presented with oculo-orofaciodigital defect with endocrinopathy and molar tooth configuration in axial magnetic resonance images (MRI). It is a rare presentation of JS.

Amita Bhargava, Gaurav Kasundra, Shubhakaran Khichar, Janardan Sharma, Banakar Basavaraj

Department of Neurology,

Dr. S.N. Medical College and M.G. Hospital, Jodhpur, Rajasthan, India

Address for the Correspondence:

Dr. Bharat Bhushan,

H. No. 21/267, Chopasani Housing Board,

Jodhpur - 340 008,

Rajasthan, India.

E-mail: drbhushan90@yahoo.com

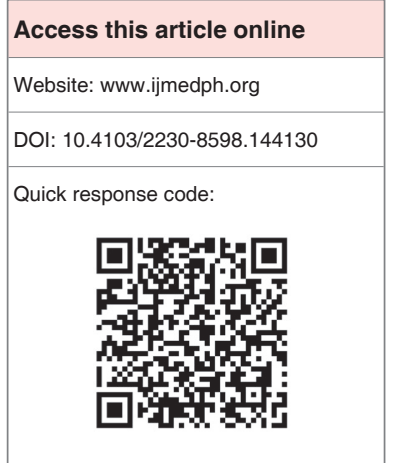

\section{CASE REPORT}

A 14-year-old boy, third born full-term delivered to nonconsanguineous parentage, presented with global developmental delay, dysmorphic feature and abnormal eye movements without significant perinatal asphyxia, seizure, abnormal breathing pattern, feeding or swallowing difficulty. First consultation was taken at age of 5 years for delayed milestone and inability to fix the eye. He was considered as static encephalopathy and never took the opinion until age 12. Two year back he went for right eye unsuccessful sling operation. Family history disclosed that out of three siblings his second born elder sister had same features. She could not achieved puebarche, thalarche and menarche until the age of 17 years. On examination, he had mild cognitive decline, ankyloglossia, right eye ptosis, postaxial polydactyly, syndactyly, pes planus, micropenis and gynecomastia [Figures 1 and 2]. He had ocular motor apraxia, inability to initiate saccades and pursuit movement. Fundus revealed retinal dystrophy [Figure 3]. Further examination revealed hypotonia, dysarthria and asymmetrical appendicular and gait ataxia. All investigations were normal including hormonal assay except low testosterone (26.2 ng/dl, normal: 40-250) level. His sister had high thyroid stimulating hormonal (16 mIU/L normal: 0.5-4.5) and low luteinizing hormone (1.5 mIU/ml, normal: 2.4-95.6), estrogen (51.3 pg/ml, normal: 61-437) levels. However, she had normal prolactine and progesterone levels. MRI of both siblings showed molar tooth configuration and bat wing fourth ventricle [Figure 4] suggestive of JS. Pituitary protocol imaging was normal in both siblings.

\section{DISCUSSION}

Diagnostic criteria for JS continue to evolve, most authors concur that the neuroradiological finding of the molar tooth sign (MTS) is obligatory. ${ }^{[1,2,4]}$ The diagnosis of "classic" or "pure" JS is based on the 


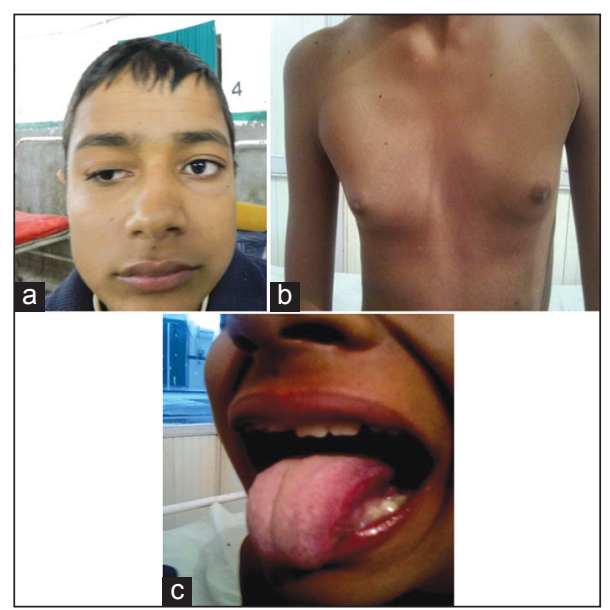

Figure 1: (a) Right eye ptosis with squint (b) gynecomastia (c) ankyloglossia

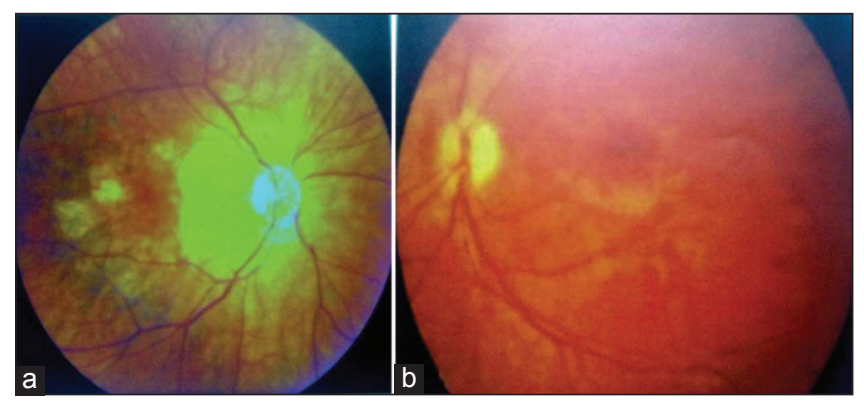

Figure 3: (a) Retinal dystrophy more diffuse in right eye (b) than left

presence of the three primary criteria: (1) The MTS. (2) Hypotonia in infancy with later development of ataxia. (3) Developmental delays/intellectual disability. ${ }^{[3]}$ Nineteen causative genes have been recognized so far, every single one encoding for proteins of the primary cilium or the centrosome have been identified in subjects Joubert syndrome and related disorder. ${ }^{[5]}$

Besides JS, cerebellar vermian anomalies have been reported with other disorders, such as Dandy-Walker syndrome and rhombencephalosynapsis. Pathological studies in these patients have shown that the cerebellar vermis is hypoplastic, and the dentate nucleus is fragmented. The ponto-mesencephalic junction is dysplastic, with abnormal decussation of the superior cerebellar peduncle and elongation of rostral fourth ventricle. There is a decrease in neurons of the basis pontis and reticular formation. In the medulla, the inferior olivary nucleus, tractus solitarius, the nucleus and spinal tracts of trigeminal nerves show evidence of hypoplasia. ${ }^{[6,7]}$

Both siblings of our case have features of JS and associated findings with the exception of breathing abnormalities which may have been overlooked. Although MTS and other important clinical features of the JS are present in both siblings, but combined features of ocular, orofaciodigital JS phenotype subgroups with endocrinopathy distinguished to previously describe six JS subgroups. Inability to achieve puebarche, thalarche and menarche in female, gynecomastia

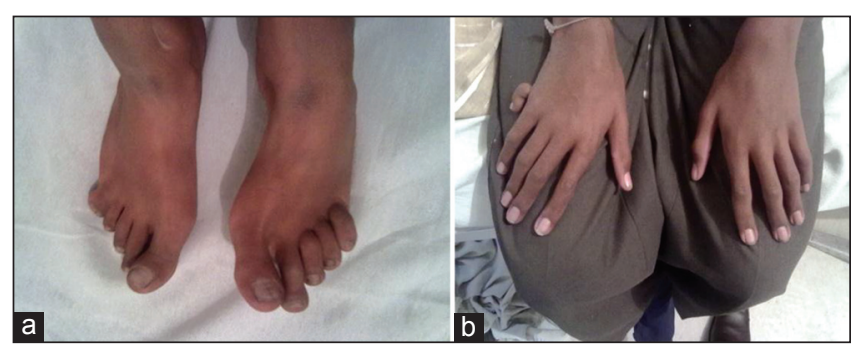

Figure 2: (a) Syndactyly in the right foot with pes planus (b) postaxial polydactyly in the right hand

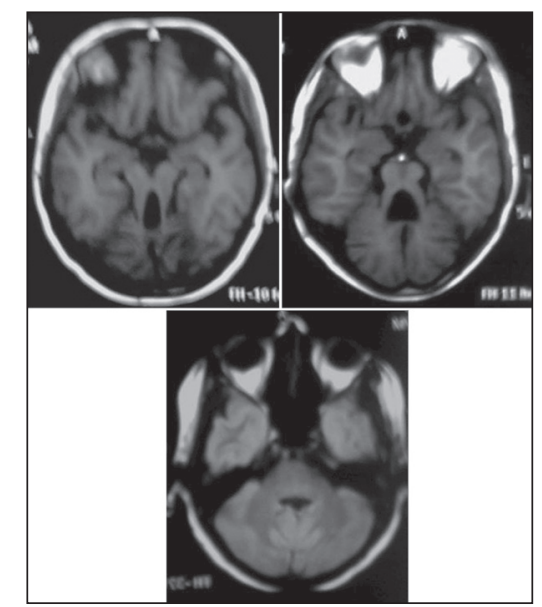

Figure 4: Axial cuts of magnetic resonance images showing "molar tooth" appearance

and micropenis in male, with their relevant hormonal disturbance and detailed phenotype consequences were described incompletely in earlier literature. Only few study mentioned endocrinopathy in JS ${ }^{[8,9]}$ Pituitary hormone dysfunction ranging from isolated growth hormone deficiency or thyroid hormone deficiency to more extensive panhypopituitarism or micropenis in males was described. ${ }^{[0]}$ Mental retardation, truncal obesity, retinal dystrophy, micropenis (MORM) syndrome and Bardet-Biedl syndrome may have similar clinical features like JS with endocrinopathy, but lacking of MTS in imaging. Individuals with MORM syndrome have normal growth parameters and life span with a congenital nonprogressive retinal dystrophy and static mild-to-moderate cognitive impairment; in contrast to Bardet-Biedl syndrome, there is no polydactyly, apparent hypogonadism, or obvious renal disease. ${ }^{[10]}$

We proposed that either combined features JS subgroup may evolve at later age with manifestation of endocrinopathy or evolving different group of JS spectrum in Indian people. Due to the limitation of sources we could not manage genetic study. The rarity of reporting to this rare syndrome is to highlight the different subgroup of JS presenting with oculo-orofaciodigital defect with endocrinopathy.

\section{ACKNOWLEDGMENT}

The authors would like to thanks Dr. Pujar S Guruprasad and Dr. Yasin Mohamad. 


\section{REFERENCES}

1. Brancati F, Dallapiccola B, Valente EM. Joubert Syndrome and related disorders. Orphanet J Rare Dis 2010;5:20.

2. Choh SA, Choh NA, Bhat SA, Jehangir M. MRI findings in Joubert syndrome. Indian J Pediatr 2009;76:231-5.

3. Egger J, Bellman MH, Ross EM, Baraitser M. Joubert-Boltshauser syndrome with polydactyly in siblings. J Neurol Neurosurg Psychiatry 1982;45:737-9.

4. Chance PF, Cavalier L, Satran D, Pellegrino JE, Koenig M, Dobyns WB. Clinical nosologic and genetic aspects of Joubert and related syndromes. J Child Neurol 1999;14:660-6.

5. Joubert M, Eisenring JJ, Robb JP, Andermann F. Familial agenesis of the cerebellar vermis. A syndrome of episodic hyperpnea, abnormal eye movements, ataxia, and retardation. Neurology 1969;19:813-25.

6. Maria BL, Quisling RG, Rosainz LC, Yachnis AT, Gitten J, Dede D, et al. Molar tooth sign in Joubert syndrome: Clinical, radiologic, and pathologic significance. J Child Neurol 1999;14:368-76.
7. Steinlin M, Schmid M, Landau K, Boltshauser E. Follow-up in children with Joubert syndrome. Neuropediatrics 1997;28:204-11.

8. Elhassanien AF, Alghaiaty HA. Joubert syndrome: Clinical and radiological characteristics of nine patients. Ann Indian Acad Neurol 2013;16:239-44.

9. Parisi MA, Doherty D, Eckert ML, Shaw DW, Ozyurek H, Aysun S, et al. AHI1 mutations cause both retinal dystrophy and renal cystic disease in Joubert syndrome. J Med Genet 2006;43:334-9.

10. Hampshire DJ, Ayub M, Springell K, Roberts E, Jafri H, Rashid Y, et al. MORM syndrome (mental retardation, truncal obesity, retinal dystrophy and micropenis), a new autosomal recessive disorder, links to 9q34. Eur J Hum Genet 2006;14:543-8.

How to cite this article: Bhushan B, Bhargava A, Kasundra G, Khichar S, Sharma J, Basavaraj B. Oculo-orofaciodigital defect with endocrinopathy in Joubert syndrome: A rare combination. Int J Med Public Health 2014;4:504-6.

Source of Support: Nil, Conflict of Interest: None declared. 